\title{
Translating Problem Statements into Actionable Solution Procedure: DeVEloping Problem-Solving SKILlS IN ChEMICAL ENGINEERING SECOND-YEAR STUdENTS
}

\author{
Louise Meunier and Nicolas Hudon \\ Chemical Engineering Department, Queen's University, Canada \\ louise.meunier@queensu.ca
}

\begin{abstract}
Junior-level chemical engineering students often struggle with solving problems in fundamental undergraduate courses. This deficiency is generally attributed, by instructors and students alike, to gaps in mathematical knowledge. However, the difficulty may instead be rooted in an inability to interpret the information from problem statements. In this contribution, a coordinated, multi-faceted instruction methodology is proposed, over a sequence of second-year chemical engineering fundamental courses, to foster the development of problem-solving strategies and to increase the confidence of students in their abilities to decipher problem data, to develop a proper mathematical framework, and to apply a first-principle approach to problem solving. Compared with traditional lectures alone, obstacles to problem solving may be overcome when students are offered additional learning streams in the form of interactive workshops and video-recorded examples. This results in raised confidence levels toward translating problems into actionable solution procedure.
\end{abstract}

Keywords: Problem-solving Skills; Solution Procedure; Chemical Engineering; Junior Level; Fundamentals Mass and Energy Balances; Situated Design Cognition Theory.

\section{INTRODUCTION}

Problem-solving is a pivotal skill developed by engineering students throughout their undergraduate studies, notably in upper years design-focused courses. Chemical engineering students typically face the first problems specific to balances of conserved quantities during their second year of study in fundamental courses, such as Introduction to Processes and Systems, Unit Operations, and Thermodynamics. The student body generally perceives these courses to be difficult even though the mathematical skills required consist in Linear Algebra and Calculus, with which students are well acquainted at that point in their education, having previously acquired experience in applying these mathematical notions, notably in first-year physics courses.

\subsection{Motivation}

Many students encounter their first significant academic obstacles during the first semester of their second year of engineering undergraduate studies. Having achieved academic success in previous years, they struggle with poor results and failing grades in these foundational courses. Consequently, their confidence in generating correct solutions is low, and their anxiety level may even prevent them from learning effectively and overcoming their difficulties. To address these issues, a didactic study was initiated in the Chemical Engineering Department at Queen's University to foster problem-solving skills in second-year students, and to increase their confidence in their ability to solve problems of increasing complexity. The project involves a sequence of two fundamental courses over two semesters. The first step was to define and understand the roadblocks that second-year students encounter and to pinpoint the essential education theory upon which instructors can formulate an effective solution strategy.

\subsection{Literature Review}

In a seminal paper published in 1958, Newell, Shaw, and Simon proposed elements of a theory of human problem solving [1]. Their aim was to establish a framework to predict the performance of a problem solver in handling specified tasks. If the steps required in problem solving were precisely defined, then they could be assessed, quantified, and ultimately coded such that a machine could achieve the same results when faced with complex problems that humans find difficult. In describing the process of problem solving, the relations between skills and results may be linked, through a series of steps, from problem definition to the communication of the final solution. More than a decade later, Simon and Newell [2] acknowledged that the original endeavour sought to demystify the human activity of problem solving. They 
likened the exercise to an inscrutable attempt at figuring out a magician's trick. Over the years, numerous studies have been published to describe approaches and steps; nevertheless, nearly half a century has since elapsed, and instructors are still struggling with the notion of effectively transmitting to students the concept of problem solving.

From Mayer [3], problem solving is defined as $a$ summary of cognitive processes focused on the change from the given state to the final state where the solution procedure is not obvious. From an engineering perspective, problem solving involves an application of theoretical knowledge through a series of processes and mechanisms to achieve a sought solution. In practice, these processes and mechanisms are often dependent on specific problems, and a systematic approach to solving general problems is not clear to engineering students seeking to develop their own solution paths in their respective field of study.

Humans are faced with problems in any and all facets of life. Through a judicious application of knowledge and processes, some problems may be overcome [4]. However, before a problem can be solved, it must be identified $[5,6]$. In the context of second-year chemical engineering courses, the problems encountered by students are easily identified and recognized as such in their textbooks and course notes. Hence the process of problem solving consists in a sequence of steps: (1) Identify the problem and read it; (2) Interpret the problem mentally and through a diagram; (3) State assumptions and develop a solution strategy; (4) Organize what is known about the problem; (5) Apply first principles to express known information into useful equations; (6) Solve and verify [6]. Similar approaches have been proposed by various educational experts over the year, for example the four phases of Polya [7], and the six operational steps of Wankat and Oreovicz, preceded by step zero: "I can" [8].

Faced with a problem, a person has a specific aim (to solve the problem) but may not know how to achieve a solution. The steps described in the previous paragraph are not intrinsically known; they must be learned and practiced. The difficulty of problem solving is accompanied by an inner uncertainty, which may give rise to tension and anxiety [9]. In a motivated student, such feelings are overlaid by curiosity, a need for new knowledge, and a search for a solution pathway [4]. A problem is relatively easy to solve when it is well defined, contains only the elements necessary and no extraneous information, and resembles other problems previously solved [10]. In chemical engineering for example, an easy problem may feature a single unit operating under stable and unchanging conditions. Conversely, dynamic problems with changing conditions in complex systems may be far more difficult to solve. The effect of time and influences of changing parameters must be incorporated into the solution procedure, which may require a numerical rather than an analytical solution [11]. The difficulty in problem solving may reside in interpreting the given information to form cognitive links between each step of the solution process.

Equipped with this knowledge, the instructor faces the intricacy of developing problem-solving abilities in juniorlevel engineering students. During their second year of undergraduate studies, these students will be shepherded across theory and examples, through which they must learn to systematically apply a solution procedure meant to bring them from problem identification to problem solution. The tasks of the instructor are to demystify the procedure and to raise the confidence level of students in overcoming the perceived mathematical obstacles.

\subsection{Problem Definition}

An undergraduate engineering class is composed of academically successful students who achieved top marks in previous years, and who typically display a high level of confidence in their ability to succeed in further studies. Some will readily apply the problem-solving methodology presented in a Processes and Systems class and continue to perform well; others will not. The first failed mid-term in the fall term of second-year engineering is often a traumatic event. Students may blame their poor math skills, their confidence may be shaken, and they (hopefully) will turn to their instructor for help in developing their problem-solving skills. Attempting to assimilate fundamental notions, students often feel lost when applying these notions to solve problems on assignments and exams. In this paper, we hypothesize that the difficulty resides in translating problem statements into actionable solution procedures.

\subsection{Selected Methodologies}

A coordinated pedagogical approach was devised and applied in a sequence of two fundamental courses taught in fall and winter terms in second year of a chemical engineering undergraduate program. The first course (Course A), entitled Chemical Processes and Systems, introduces fundamental concepts for chemical engineering analysis, including systems of units, and mass and energy balances for chemical processes. During the fall term, when Course A was delivered, student feedback was collected after the $5^{\text {th }}$ and $11^{\text {th }}$ week of the term, as well as through ad-hoc discussions. Students evaluations were administered through university services in the final week of the term. The second course (Course B), entitled Thermodynamics of Energy Conversion Systems, introduces the students to the first and second laws of thermodynamics, as well as to the concepts of efficiencies. The course is organized around applications of thermodynamics in solving energy systems via the solution of mass, energy, and entropy balances. Essentially, Course $\mathrm{B}$ reinforces concepts and methods from Course A. During the first week of Course B, students were surveyed on their 
confidence and perception of problem-solving, and final feedback on some of the actions taken to improve course material was obtained at the end of the term. Faculty involved in both terms applied a problem-based learning methodology, involving a detailed solution procedure presented systematically, as suggested by Fredrickson [12]. Adjustments to the pedagogical approach were made based on student response to survey, student evaluations, and feedback.

In response to informal early feedback from students enrolled in Course A, further emphasis was placed on solving balance equations throughout all instructional activities. Notably, as part of an effort to integrate mathematical computing software (MCS) into problemsolving strategies, two assignments were devised for the students to develop the proper mathematical framework and to solve numerically using MCS. To reinforce the problem-solving strategy, both assignments were supported by carefully structured tutorials and supplemental examples, provided on a just-in-time basis. Generally, students adhered to the proposed method and were successful in solving the assigned problems using all available resources.

The incoming students survey proposed to the students at the beginning of Course $\mathrm{B}$ focused on two points: confidence and habits in problem-solving, and exam preparation habits. Results showed that students are generally confident in their problem-solving skills, whereas deriving the proper mathematical framework and linking theory to application are perceived as the most difficult tasks when tackling a problem.

A general problem-solving procedure, adapted from Polya's list [7], was first presented and illustrated. However, in response to survey results, the focus of all problem-solving activities was shifted. As such, establishing the proper general mathematical framework in the form of mass, energy, and entropy balances - became the focal point in all course activities, including in-class examples, video examples, and tutorial demonstrations. The framework was also reinforced in the problem formulation, marking scheme, and provided solutions for all assignment problems, supplementary problems, and exam problems. The objective was to demystify the mathematical content, which is consistent throughout the course, so that the students focus on linking theoretical contents through problem context and transform any problem into an actionable set of problem-solving steps. Early results showed improvements in students' problemsolving activities.

\subsection{Significance of the Results}

This study contributed to an increased awareness among instructors about the nature of the obstacles faced by second-year chemical engineering students. Having pinpointed the issue of translating problem statements into actionable solution procedure helped instructors to coordinate a consistent approach in teaching the steps required to generate a comprehensive and correct solution. The results of this study will serve as a benchmark to monitor student and instructor performance moving forward. The long-term objective of this recently initiated project is two-fold: first, to establish a consistent problemsolving approach to be implemented throughout the "theoretical spine", i.e., for all fundamental courses in the chemical engineering curriculum; and, second, to foster students' ownership of their learning process by promoting an active-learning environment centered on problemsolving methodologies adapted to chemical engineering.

This contribution is structured as follows. In Section 2, early observations on problem-solving are presented, as perceived by the students, and a conceptual framework is described to translate into specific actions the instruction of chemical engineering fundamental notions over a sequence of two second-year courses. In Section 3, preliminary results and observations gathered during the first implementation of the proposed problem-solving instruction approach are examined. Areas for future investigations within second year as well as upper-year courses in the curriculum are also discussed.

\section{PROBLEM SOLVING FOR JUNIOR-LEVEL CHEMICAL ENGINEERING STUDENTS}

At Queen's University, all first-year engineering students are registered in a core program. During this first year, they select their field of study, and are admitted to a specific engineering department at the beginning of their second year. Thus, chemical engineering undergraduate students are first exposed to chemical engineering problems and specific problem-solving methodology in the second-year fall-term Chemical Process and Systems class (Course A), although it must be noted that similar courses (i.e. Material and Energy Balances) are taught during first years in some universities. Course A introduces the notions of mass and energy balances pertaining to chemical processes of growing complexity (from the analysis of a single process unit at steady-state to the analysis of multiple interconnected units evolving with respect to time). These notions are the starting point of Course B, Thermodynamics of Energy Conversion Systems, in which fundamental notions from the first and second laws of thermodynamics are attached to mass, energy, and entropy balances. The fundamental notion of balances (or, in upper-year courses, the notion of conservation laws) is central to all problems of chemical engineering. This key message must be delivered early in the curriculum, and Courses A and B are logical avenues where problemsolving methodologies are applied to problems typical of chemical engineering. 


\subsection{Classroom Observations}

This engineering education project is motivated, in part, from behaviours observed by the authors in the classroom, as well as from discussions with colleagues on students' abilities to solve chemical engineering problems. One key observation, made by most instructors, pertains to tutorial sessions, where problems are posed and solved in front of the class. These sessions are generally led by graduate teaching assistants (TAs). In most cases, to foster active learning, a problem is broken into clear steps with allotted time for the students to progress sequentially toward the solution. In contrast with "traditional" learning, in which students are passive recipients of knowledge, active learning aims to engage students in activities requiring talking, writing, problem-solving and reflecting [13]. However, despite this "active" format, it has been observed that students stand passively, waiting for the solution to be developed on the board. Students appear not to have the desire or the confidence to even begin to develop a solution on their own.

Instructors also observed that students usually perform better in courses where exams dictate explicit solution procedures (with parts $a, b, c, \ldots$ ), when compared with "open" question formulations. Generally, if students are asked explicitly to draw a diagram, to state assumptions, etc., they perform well in solving the entire problem. Otherwise, some students do not apply the solution procedure, do not perform the required steps, and may try to solve the problem ad hoc. In the latter case, the success rate drops dramatically. The resulting failure is often attributed to students' lack of mathematical abilities; but this may only be a symptom of the true problem rather than its root.

We posit that the general issue with problem-solving, at least for non-experienced or junior students, is the lack of a systematic methodology to translate given problems into actionable solution steps. For such a method to be adopted by students, it must be general and flexible enough to accommodate different classes of problems, i.e., it should be a method that students can trust in solving assignment problems, in preparing for exams, and in solving exam problems. To ensure continuity between courses throughout the curriculum, the key issue was thus to identify a key concept common to at least a majority of fundamental courses. In chemical engineering, the concept of balance laws (or more generally, conservation laws) serves that purpose, as detailed in the next section.

\subsection{Conceptual Framework}

Introducing a systematic problem-solving methodology to junior-level chemical engineering students for a sequence of two courses required the identification of a common conceptual framework. This structure was elaborated following in-class observations as well as a review of observations from authors and users of learning theories, including Problem-Solving [1, 5], Active Learning [13], Experiential Learning [14], and Problembased Learning [12]. Specifically, the method must be simple and directly applicable to typical problems which students must solve $[1,5]$. For students to effectively adopt a problem-solving methodology, an explicit and methodical approach to the solution is required, including extensive scaffolding and guidance [12]. The rationale for this approach must be explicitly stated to motivate students toward implementing and trusting the proposed methodology [13], and the selected problems must be relatable, drawing on experiences and issues that students have or may soon encounter [14].

As mentioned above, a key element common to all chemical engineering courses requires the application (and solution) of balance laws or, more generally, of conservation laws. Equipped with differential and integral calculus and linear algebra (taught in first year courses), junior-level engineering students can solve these problems. Although the nature of chemical engineering balance problems differs somewhat from first-year physics and mechanics, the mathematical tools are similar.

Organizing Course A around the concept of balance laws is natural because the fundamentals of mass and energy balances form the core of the curriculum [15]. However, this is a not common approach in Course B, except in the textbook [16]. To ensure continuity between both courses, and to reinforce the selected methodology, the concepts of balances was implemented as the backbone of Course B.

The purpose of this approach was to demonstrate the generality of balance laws as a concept applicable to all problems, in both courses and beyond. This was achieved by deconstructing problems following the problem-solving map depicted in Figure 1. This map was applied to all problem activities (in-class examples, tutorials, assignment solutions, and video examples), reinforcing a general problem-solving approach, where inputs are taken from problem data, assumptions are implemented for problemspecific circumstances, and theory is viewed through the central objective of solving balance equations. Variations on problems (extensions) are also presented from the perspective of balance laws. As a tool to develop material for Course B, the conceptual map proved to be practical and improved efficiency in material delivery. 


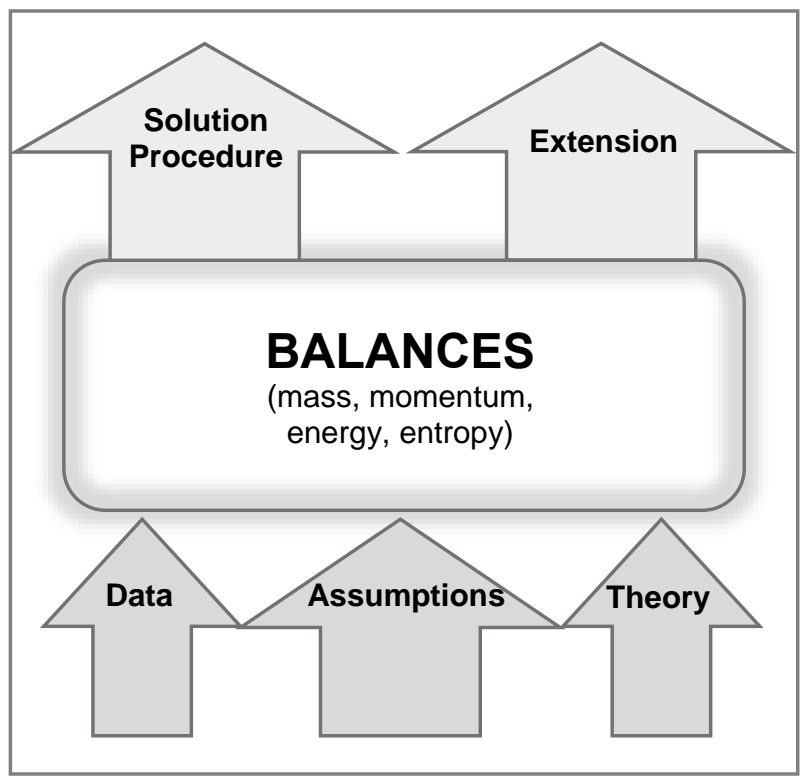

Figure 1: Problem-solving methodology centered on balance laws.

\subsection{Assessing Abilities and Confidence}

At the beginning of Course B, students where asked to complete a survey on their confidence in their own problem-solving abilities, and on their exam preparation. A total of 73 students responded to the survey, and selected results are presented here. Analysis of these results triggered some modifications to the instruction of Course B.

Generally, more than $75 \%$ of the students acknowledged that they have been exposed to some form of problem-solving approach as early as high school. For most students, these concepts were refined in their first year of engineering education. As a result, when asked "Do you feel confident in your problem-solving skills?" on a scale ranging from 0 (not at all) to 5 (very confident), the majority feels "Generally Confident". The distribution is reported in Figure 2.

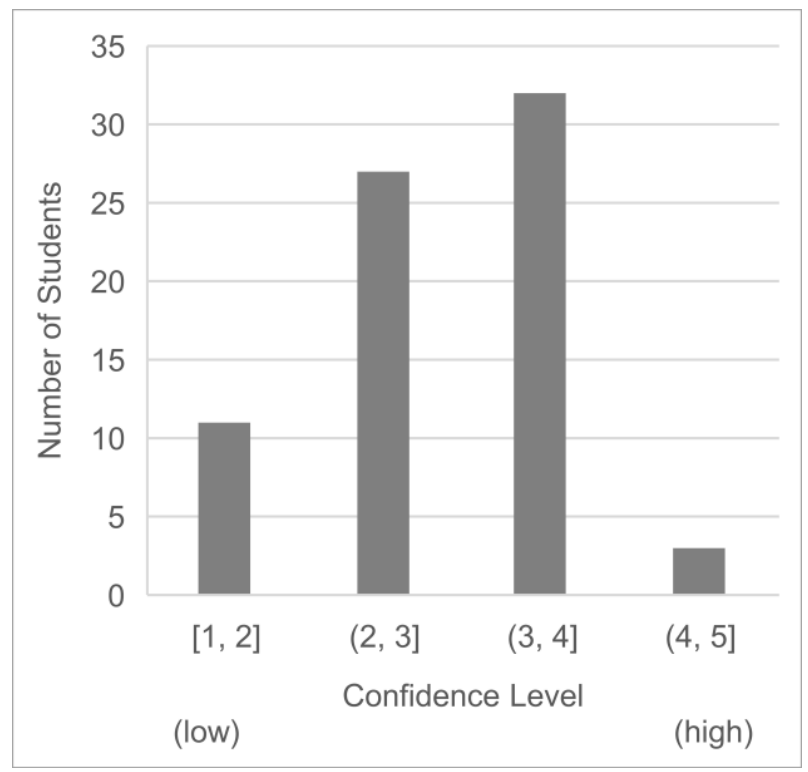

Figure 2: Students confidence in their problem-solving skills $(\mathrm{N}=73)$.

Presented with a list of problem-solving steps, students where asked to assess which are the easiest and the most difficult. The steps were defined as follows:

1. Read the problem;

2. Connect problem to theory;

3. Make a diagram/picture of the problem;

4. State assumptions;

5. Develop mathematical equations;

6. Find values in tables/charts;

7. Perform computations;

8. Check numerical answer/unit consistency;

9. Other.

In response to this question, students identified "Read the problem", "Find the values in tables/charts", and "Perform computations" as the easiest perceived steps. The distribution of the answers (out of a maximum of 1 ) is reported in Figure 3. 


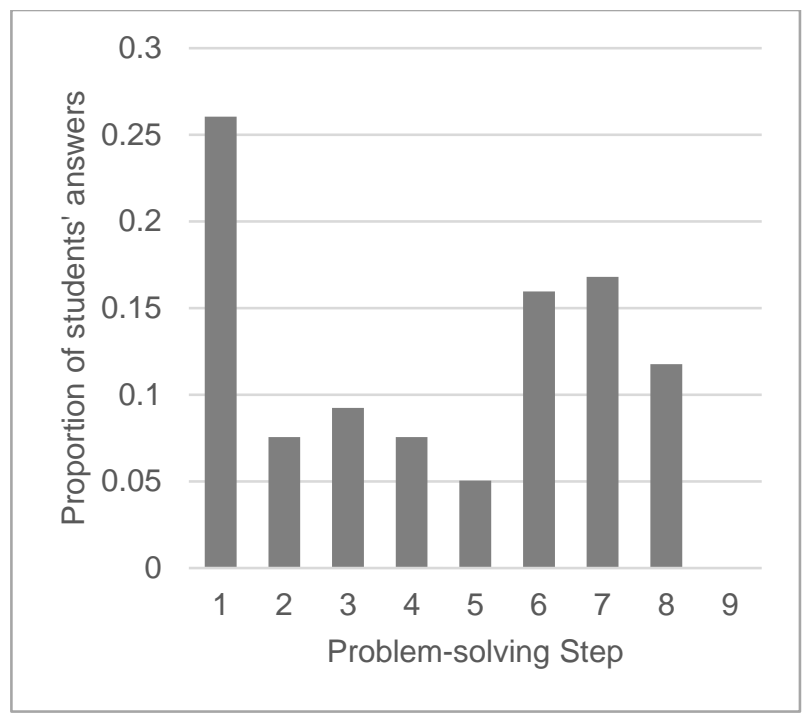

Figure 3: Distribution of student responses to their perceived "Easiest step" in problem solving.

When asked "Which of the following steps is the most difficult for you when solving a problem?", students identified "Develop Mathematical Equations" and "Connect Problem to Theory" as the tasks perceived to be most challenging in problem solving. The distribution of student answers is reported in Figure 4.

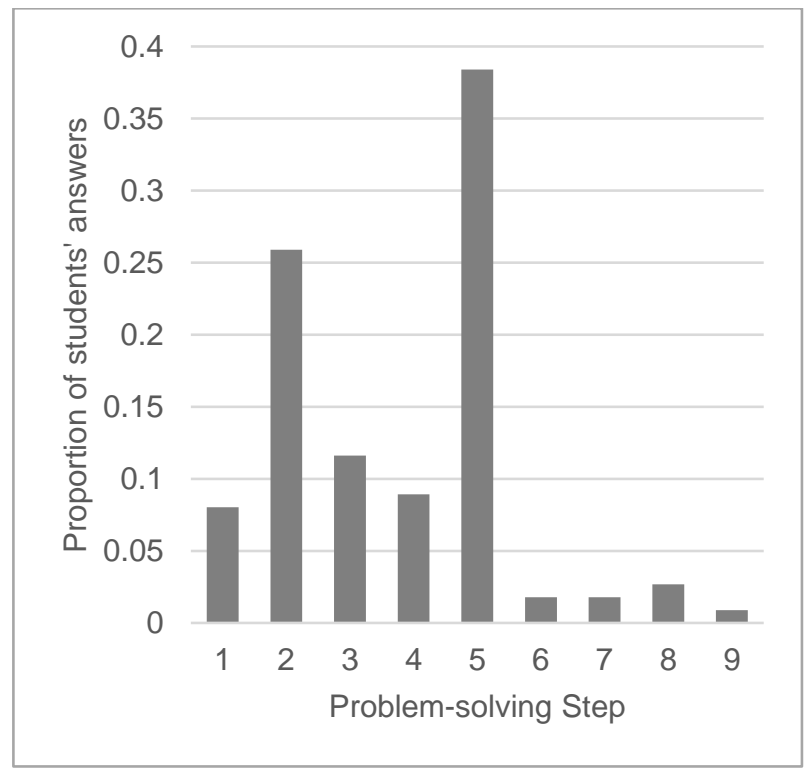

Figure 4: Distribution of student responses to their perceived "Most difficult step" in problem solving.

The results appear, at first glance, to contradict the hypothesis stated above, because results seem to suggest that deficiencies in mathematical skills are indeed the root cause of problem-solving deficiencies for junior-level chemical engineering students. However, we argue that the results reflect the students' own perceptions and not the reality. The results of the survey were nonetheless useful, because they underlined the necessity of demystifying the mathematical framework, which is the purpose of the concept map depicted in Figure 1, and this became the focal point of problem-solving demonstrations and activities. In Course B, the emphasis was shifted from scaffolding the early stages of problem-solving strategies (identifying the question, identifying the data, etc.), as suggested, for example, by Polya's list [7], to the construction of the central mathematical framework (the balance laws), written first in a general form, and then simplified and adapted through problem statement analysis. Early observations of student performance suggest an improvement in their problem-solving confidence in midterm exams and their willingness to apply the problemsolving methodology systematically in assignments, tutorial problems, and tests. In other words, by demonstrating how to interpret problem systematically through the development and solution of balance laws, i.e., by organizing problem-solving demonstrations following the map proposed in Figure 1, mathematical difficulties and students' anxiety were apparently alleviated.

\subsection{Selected Methodologies in Course A}

In Course A, a thoroughly systematic approach is presented to the students. The material is developed in six modules - each building on the previous one - starting from a simple mass balance in a single unit at steady-state and ending with simultaneous mass and energy balances for multiple units in variable conditions. Emphasis is placed on solution procedure and methodology. Full solutions are presented in lecture notes, on the blackboard, in problem sets, and during interactive tutorials. Course material is available in printout form (course reader) and through an online learning platform in an incomplete format that students complement during lectures and workshops. Students are also invited to extend their problem-solving skills in numerical methods (MCS assignments). Multiple assessments (weekly online reading and comprehension assignments, seven written assignments, three quizzes, one final exam) are scheduled throughout the term to help students gauge their progress. Tests incorporate the methodology presented in class and illustrated in Figure 1. Student feedback is collected throughout the term and both instructors coordinate their approach to follow the same methodology and to ensure a coherent approach in the classroom. It should be noted that the instructor of Course B was involved in the development of MCS-related assignment problems, tutorials, and extended examples used in Course A. In the development of this set of specific material, the approach outlined above, and illustrated in the Problem-solving methodology depicted in Figure 1, was systematically employed. 


\subsection{Selected Methodologies in Course B}

Building on notions introduced and developed in Course A, Course B introduces fundamental elements of classical thermodynamics. Conceptual consequences and applications of the first and second laws of thermodynamics, are integrated throughout the term by solving problems of increasing complexity through the solution of balance laws, which is reviewed during the first week of instruction. The general problem-solving strategy is systematically employed in in-class examples, tutorials, assignment solutions, and video examples. The positive impact of the latter has been demonstrated, as students appreciate this opportunity to follow along, and to review concepts as they draft problem solutions and assignments, and in preparation for exams. Videos enable the instructor to clearly identify and reinforce the proposed problemsolving strategy and to present a clear method summary, all of which is presented freely, without the constraint of limited lecture time. In addition, students can pause and re-watch the videos at their leisure, which helps them to understand the problem-solving approach.

In course B, students are required to complete weekly assignments (twelve in total), and a set of supplementary problems is suggested. Solutions to all these problems are available to the students, and all solutions are developed following the proposed problem-solving procedure centered on balance laws.

Both quizzes and the final exam for Course B are designed according to the general problem-solving methodology depicted in Figure 1, i.e., exam problems are developed through an explicit sequence of sub-questions developed according to the problem-solving strategy. However, in each exam, one question is posed in a free form. It has been observed that a majority of students apply the full problem-solving procedure even if they are not explicitly asked to draw a schematic, develop balance equations, and state assumptions, etc.

\section{RESULTS AND DISCUSSION}

In this article, we report some preliminary results and observations collected at early stage of this engineering education project. The objectives, over the next few years are to refine the integrated problem-solving educational approach, and to collect results to monitor progress. In particular, an exit survey of students' problem-solving confidence was conducted at the end of Course B Winter 2019 term. Early results and observations show improvements in students' confidence in their ability to successfully complete marked activities. Conversely, poor student engagement is noted as an issue that should be addressed in future years.

\subsection{Results and Observations from Course A}

Students generally completed their work on time, and approximately $70 \%$ of students attended additional tutorial time at least once per week. More than $90 \%$ of students completed the online reading assignments and comprehensive questions, and more than $65 \%$ of students attempted to solve all suggested questions from the problem sets. Among the group of 98 students enrolled in Course A for the Fall 2018 term, 6 requested additional practice problems. These were posted online, along with detailed solutions. The average marks on quizzes ranged from $\mathrm{C}$ - to $\mathrm{C}+$; average failures ranged from 12 to $26 \%$ of the group, and 9 to $22 \%$ of students achieved marks of Aof better. The final average mark for this course was a $\mathrm{C}+$; 16 students failed to achieve a passing grade, and 15 students obtained a mark of A- or better.

Failures are generally associated with difficulties in setting up a correct process flow diagram and in an inability to produce an accurate mass and/or energy balance. Comments from the course evaluation included expressions of anxiety and lack of confidence.

\subsection{Results and Observations from Course B}

Results for Course B, which is taught during the Winter 2019 term, are incomplete. However, comparing results for the first four assignments to those from previous terms, grades are generally up. Informal discussions with students during office hours also suggest that confidence levels (prior to exams) are generally higher than in former years.

In general, it seems that students understand the course expectations, and have a good idea of how to proceed with the proposed problem-solving methodology to demonstrate their understanding of concepts on exams, which appears to be an improvement over the perceptions from students in previous years. Instructors in both Course A and Course B noted that students typically complain less about "mathematical difficulties" than in past years.

Failures (in assignments and exams) generally coincide with a lack of engagement in the course, i.e., students who fail assignments typically do not attend lectures and/or tutorials. The main difficulty for the instructor of Course $\mathrm{B}$ at this point is to motivate the students to go beyond a passive behavior in the course, i.e., to avoid relying solely on video examples and published problem solutions.

\section{CONCLUSIONS}

This paper presents early approaches to improve problem-solving skills of junior-level chemical engineering students at Queen's University. The strategy proposes to develop a continuous and consistent approach for a sequence of two fundamental courses in the curriculum, which aims to help students increase their 
confidence and their ability to solve general problems in chemical engineering. The proposed hypothesis posited that the main difficulty faced by students reside in translating a problem into actionable steps, rather than in overcoming mathematical deficiencies. This requires initiating a shift in the perception that students have of the most difficult steps in problem solving. Indeed, survey results indicated that students perceive mathematical content as their main difficulty when solving problems. The focus of instruction in both courses was to apply a clear methodology in an effort to demystify chemical engineering problem solving. Based on problem-solving and learning theories, a generic methodology was proposed, where balance laws are central to the problemsolving process. Early results and observations indicate that students engaged in the approach are more confident and successful in problem-solving. Further data will be collected in coming years to evaluate the effectiveness of the proposed approach.

Key elements were also identified for future years. First, getting students engaged in problem-solving is essential, as the overall goal is not for the students to learn and apply a given methodology, but rather to adapt and expand on given methodologies to develop their own approach to problems. To this end, tutorial delivery will be modified to an interactive format to promote students' ownership of learning. Second, for the proposed approach to be successful in developing engineering graduates that are confident and efficient in solving problems of growing complexity, the current study will be expanded to upperyear fundamental courses of instruction. Both the proposed methodology and the desired students' behaviour in problem-solving approach is to be promoted and incorporated throughout the chemical engineering curriculum over the next few years.

\section{Acknowledgements}

The authors acknowledge funding support from the National Science and Engineering Research Council (Discovery grants for the first author) and from the Faculty of Engineering and Applied Science at Queen's University for both authors.

\section{References}

[1] Newell, A., Shaw, J.C., and Simon, H.A. 1958. "Elements of a Theory of Human Problem Solving", Psychological Review, 65:151-166.

[2] Simon, H.A. and Newell, Al. 1971. "Human Problem Solving: The State of the Theory in 1970", American Psychologist, 26(2):145-159.
[3] Mayer, R.E. 1990. "Problem solving", In W.M. Eysenck (Ed.): The Blackwell Dictionary of Cognitive Psychology, Basil Blackwell, Oxford, 284-288.

[4] Dostal, J. 2015. "Theory of Problem Solving”, Procedia Social and Behavioral Sciences. 174: 2798-2905.

[5] Mayer, R.E., and Wittrock, M.C. 1996. "Problem-solving Transfer", in D. C. Berliner and R. C. Calfee (Eds): Handbook of Educational Psychology. Simon \& Schuster Macmillan, New York, 47-62.

[6] Pretz, J.E., Naples, A.J. and Sternberg, R.J. 2003. "Recognizing, Defining, and Representing Problems", in J.E. Davidson and R.J. Sternberg (Eds): The Psychology of Problem Solving. Cambridge University Press, UK, 1-30.

[7] Polya, G. 2004. How to Solve It. Princeton University Press. $253 \mathrm{p}$.

[8] Wankat, P.C. and Oreovicz. F.S. 2015. Teaching Engineering, $2^{\text {nd }}$ Ed. Purdue University Press, USA. 450 p.

[9] Duncker, K. 1945. "On Problem Solving”, Psychological Monographs, 58(5): i-113

[10] Mayer, R.E., and Wittrock, M.C. 2006. "Problem solving", in P.A. Alexander and P.H. Winne (Eds): Handbook of Educational Psychology, Laurence Erlbaum Associates, Mahwah, New Jersey, 287-303.

[11] Wirth, J. and Klieme, E. 2003. "Computer-based Assessment of Problem-Solving Competence", Assessment in Education: Principles, Policy \& Practice. 10(3):329345 .

[12] Fredrickson, R. McMahan, S. and Dunlap, K. 2013. "Problem-Based Learning Theory", in B.J. Irby, G. Brown, R. Lara-Alecio, and S. Jackson (Eds): The Handbook of Educational Theories. Information Age Publishing, USA, 211-217.

[13] Centre for Educational Innovation. Office of the Executive Vice President and Provost, University of Minnesota. Active Learning. Available as of February 14, 2019 from https://cei.umn.edu/active-learning.

[14] Zijdemans-Boudreau, A. Moss, G. and Lee, C.G. 2013. "Experiential Learning Theory", in B.J. Irby, G. Brown, R. Lara-Alecio, and S. Jackson (Eds): The Handbook of Educational Theories. Information Age Publishing, USA, 115-123.

[15] Felder, R.M. Rousseau, R. W. and Bullard, L.G. 2016. Elementary Principles of Chemical Processes, $4^{\text {th }}$ Ed. Wiley, New York. 674p.

[16] Sandler, S.I. 2006. Chemical, Biochemical, and Engineering Thermodynamics, 4th Ed. Wiley, New York. 960p. 